\title{
Typhoid fever among febrile Nigerian patients: Prevalence, diagnostic performance of the Widal test and antibiotic multi-drug resistance
}

\section{Martin E. Ohanu1, Michael O. Iroezindu², Uzoamaka Maduakor ${ }^{3}$, Obinna D. Onodugo ${ }^{2}$, Harish C. Gugnani}

1. Department of Medical Microbiology, College of Medicine, University of Nigeria, Ituku/Ozalla, Enugu, Nigeria

2. Department of Medicine, College of Medicine, University of Nigeria, Ituku/Ozalla, Enugu, Nigeria

3. Department of Medical Laboratory Sciences, Faculty of Health Sciences and Technology, University of Nigeria, Enugu Campus, Enugu, Nigeria 4. Department of Microbiology, Vallabhbhai Patel Chest Institute, University of Delhi, Delhi, India

\begin{abstract}
Background
Abstract

Over-dependence on clinical presentation and/or the Widal agglutination test for the diagnosis of typhoid fever in developing countries can lead to antibiotic abuse. In Nigeria, the antibiotic resistance of typhoid organisms is poorly characterized. In this study, we determined the prevalence of culture positivity among patients suspected of having typhoid fever, evaluated the diagnostic value of the Widal test and the burden created by the multi-drug resistance of typhoid organisms in South-East Nigeria.

Methodology

This was a prospective and case-controlled study carried out between 2013 and 2016. We acquired samples of blood/stool/urine cultures, and data relating to the Widal agglutination test and malaria parasites from 810 febrile patients (suspected of having typhoid) and 288 apparently healthy controls. Individuals with a history of antibiotic use within the previous 14 days were excluded. We then carried out antibiotic susceptibility tests on all isolates. Multi-drug resistance was defined as a resistance to $\geq 3$ of the antibiotics tested. We determined the sensitivity, specificity, positive predictive value (PPV) and negative predictive value (NPV) of Widal test for typhoid laboratory diagnosis compared to bacterial culture which is the gold standard. A P-value $<0.05$ was considered to be statistically significant.

Results

The mean age of typhoid suspects was $33.1 \pm 6.5$ years and $50.7 \%$ were women. Of the 810 typhoid suspects tested, $114(14.1 \%)$ had positive cultures for the typhoid organisms Salmonella enterica serovar paratyphi (72) and S. enterica serovar Typhi (42). Sample-specific rates of culture positivity were as follows: stool $(72 ; 8.9 \%)$, blood $(21 ; 2.6 \%)$ and urine $(21 ; 2.6 \%), \mathrm{P}<0.001$. None of the controls had typhoid isolates. The sensitivity, specificity, PPV and NPV of the Widal test were $49.1 \%, 90.7 \%, 46.2 \%$ and $91.6 \%$, respectively. Malaria parasitaemia was detected in $180(22.2 \%)$ febrile patients, out of whom $115(63.9 \%)$ had a positive Widal test for O/H antigens vs. $1 \%(6 / 630)$ in those with negative malaria parasite test results $(\mathrm{P}<0.001)$. The rate of false-positive Widal titres was $48 \%$. Antibiotic multi-drug resistance was detected in $52.6 \%$ of patients. The antibiotics with the highest susceptibility were ciprofloxacin, levofloxacin and meropenem (all 100\% susceptibility) and ceftriaxone (95.6\% susceptibility).

Conclusion

Our data showed that while typhoid fever is common in Nigeria, malaria is more prevalent. Our analysis showed that the Widal test performed poorly as a diagnostic test and that the burden created by multi-drug resistance was high. Our data indicate that periodic surveillance of antibiotic susceptibility is critical for optimal typhoid therapy.
\end{abstract}

Key Words

Antibiotics, diagnosis, typhoid, malaria, multi-drug resistance, Widal test

\section{Introduction}

Typhoid fever is an acute and sometimes life-threatening systemic febrile illness caused by Salmonella enterica serovar Typhi (S. typhi) and S. enterica serovar paratyphi (S. paratyphi) A, B or C. Although the disease caused by $S$. paratyphi has traditionally been thought to run a more benign course ${ }^{1,2}$, recent observations have indicated that $S$. paratyphi has an almost identical clinical syndrome to $S$. typhi $i^{3}$.Typhoid is transmitted by water and food which are contaminated by faeces; human beings are the only known reservoir. Typhoid remains a global public health problem with a higher burden in low- and middle-income countries (LMICs) due to poverty, limited access to safe water and unhygienic practices ${ }^{4}$. In 2000, there were 21.6 million new cases of typhoid fever, 210,000 typhoid fever-related deaths and 5.4 million cases of paratyphoid fever ${ }^{5}$. In 2010, Buckle et al. estimated that there were 13.9-26.9 million cases of typhoid fever worldwide ${ }^{6}$. Without effective treatment, typhoid fever is associated with a case-fatality rate of $10-30 \%$, although this reduces to $1-4 \%$ in those receiving appropriate therapy ${ }^{5}$. Only a limited amount of data are available for the burden created by typhoid in several LMICs. Consequently, there is growing interest in carrying out studies of disease burden created by typhoid fever in these settings ${ }^{7-9}$. The Typhoid Fever Surveillance in Africa Program (TSAP) was established by the International 
Vaccine Institute to acquire comparable incidence data on typhoid fever and invasive non-typhoidal Salmonella (iNTS) disease in sub-Saharan Africa. This program adopted standardized surveillance protocols in a number of different countries, including Burkina Faso, Ethiopia, Ghana, GuineaBissau, Kenya, Madagascar, Senegal, South Africa, Sudan and Tanzania ${ }^{7}$. Each TSAP site carried out case detection using standardized methods to isolate and identify aerobic bacteria from the bloodstream of febrile patients. Incidentally, despite being one of the most populous countries in Africa, Nigeria is not among the TSAP sentinel countries. Using a mixed-effects model, which was fitted to data originating from 32 population-based studies of typhoid incidence in 14 countries (including some TSAP sentinel countries), Antillon et al. previously estimated that 17.8 million cases of typhoid fever occur each year in LMICs. Moreover, Central Africa was predicted to experience the highest incidence of typhoid, followed by certain countries in Central, South, and Southeast Asia ${ }^{8}$.

The isolation of $S$. typhi from blood or bone marrow is considered to represent the gold standard for the diagnosis of typhoid ${ }^{10}$. Although stools and urine are not sterile sites, and but considering blood culture is often unsuccessful, the isolation of $S$. typhi from urine or stools remains relevant in the diagnosis of typhoid, particularly in individuals with compatible clinical features. However, one must consider that the culture of such samples requires laboratory equipment and expertise that are not readily available in most primary health centres in resource-limited settings ${ }^{11}$. On the other hand, the Widal agglutination test is relatively affordable, easy to perform and requires minimal equipment or expertise. However, the Widal test has several limitations ${ }^{12-15}$. For example, Widal test cannot distinguish between a current infection and a previous infection or vaccination against typhoid. Widal test shows cross-reactivity with other Salmonella species. Physicians in developing countries are often faced with the challenge of making treatment decisions on the basis of compatible clinical symptoms alone or a combination of clinical symptoms and Widal results obtained from a single acute-phase sample ${ }^{16-19}$. These factors lead to high rates of inaccurate typhoid diagnosis, inappropriate antibiotic therapy, considerable antibiotic multidrug resistance (MDR) and potentially worse outcomes ${ }^{19}$. Despite the growing concern related to antibiotic MDR in the management of typhoid fever, only a few studies have assessed the current burden created by this disease in subSaharan Africa. We determined the prevalence of typhoid fever (as confirmed by culture) among patients presenting with acute febrile illness in a major referral hospital in SouthEast Nigeria. We also assessed the diagnostic value of the Widal test using single acute-phase samples and determined the pattern of antibiotic susceptibility pattern and MDR in typhoid isolates.

\section{Methods}

\section{Study design/study area}

This was a prospective case-controlled study conducted at the University of Nigeria Teaching Hospital (UNTH), Ituku/Ozalla, Enugu. The UNTH is the largest referral centre in South-East Nigeria. In 2014, the estimated population of Enugu state was $4,139,598^{20}$ with a population density of approximately 460 people per square kilometre. Furthermore, approximately $47.5 \%$ of households in Enugu state have improved sources of drinking water, while only
$19 \%$ have improved sanitary facilities that are not shared by more than one family ${ }^{21}$.

\section{Study population}

We recruited patients between 1 June 2013 and 31 May 2016 attending the outpatients clinic at UNTH. We recruited a total of 810 consecutive typhoid fever suspects (141 children aged $<18$ years and 669 adults $\geq 18$ years) presenting with fever (axillary temperature $>37.5^{\circ} \mathrm{C}$ ) and any two of our inclusion criteria (abdominal pain or discomfort, headache, constipation or diarrhoea). We focused on body temperature in our inclusion criteria (rather than the history of fever, which is largely subjective) in order to make our blood culture surveillance more sensitive. During the same period, 288 apparently healthy individuals (48 children $<18$ years and 240 adults $\geq 18$ years), who were mostly the relatives of the patients, were recruited as controls and were matched with the patients for age and gender. Individuals who had received antibiotics within the previous 14 days were excluded; this limited the impact of prior antibiotic use on our culture results.

\section{Ethical considerations}

Ethics approval was obtained from the Health Research and Ethics Committee of the UNTH (Reference number: UNTH/CSA/329/VOL.5; Registration number: NHREC/05/01/2008B-FWA 00002458-IRB 2323). Written (signed) informed consent was obtained from each participant before enrolment and confidentiality was assured.

\section{Sample collection and cultures}

Prior to the administration of antibiotics, we collected blood, urine and stool samples from each of the 810 typhoid suspects for culture. Blood, urine and stool cultures were also processed for the 288 controls. Blood cultures were performed by aseptically introducing $10 \mathrm{ml}$ of blood sample into brain heart infusion and thioglycolate-containing liquoid media, as described previously by Baron and Finegold ${ }^{22}$. Subcultures from positive blood cultures were performed using blood agar and storing them as stock until the isolates were completely identified as typhoid organisms.

Approximately $1 \mathrm{~g}$ of stool sample from each participant was suspended in $5 \mathrm{ml}$ of sterile saline solution and inoculated into appropriate types of bacteriological agar before and after enrichment with selenite-F broth. Specimen and subcultures of stools were plated onto MacConkey and deoxycholate agars (Oxoid) and incubated for 24-48hours at $37^{\circ} \mathrm{C}$. The resultant suspect colony growths were examined for Salmonella using standard methods ${ }^{23}$.

Fresh colonies are non-lactose fermenting, circular, smooth, glossy, and translucent with delicate nature. $S$ typhi ferments maltose, glucose and manitol producing acid but no gas. It reduces sulphide in triple sugar iron agar to produce a black colour. Urease test was performed to rule out Proteus spp among others. Suspected isolates were subjected to rigorous serological identification to species level after performing a preliminary identification with polyvalent Salmonella $O$ antiserum (Oxoid). Slide agglutination was first used and positive results were confirmed by tube tests ${ }^{23}$.

For each participant, $10 \mathrm{ml}$ of urine was centrifuged at 3000 rpm for 15 minutes and the resulting sediment inoculated onto cysteine lactose electrolyte deficient (CLED) agar before and after enrichment with selenite-F broth. The resultant suspect colony growths were examined for Salmonella by 
standard methods ${ }^{23}$

During the processing/analysis of samples, we used relevant standard operating procedures (SOPs) of the Microbiology Department such as SOP for specimen collection, transportation and processing, SOP for microbiological safety practices, SOP for processing of blood/other sample cultures, Culture media quality control SOP and SOP for antimicrobial susceptibility testing. All instruments were periodically checked and calibrated by hospital biomedical engineers.

\section{Widal agglutination test}

Sera samples from each of the 810 typhoid suspects and 288 controls were used to carry out a semi-quantitative Widal agglutination test; this was performed using the standard tube dilution procedure ${ }^{24}$ and Salmonella antigen kits manufactured by Gamma Biologicals Inc. (Houston, TX, USA). In accordance with the manufacturer's protocol, we used an antibody titre of $\geq 1: 80$ for $\mathrm{O}$ antigen, and a titre of $\geq 1: 160$ for $H$ antigen, as cut-off values for positive titres. A negative saline control was used for each batch of Widal tests.

\section{Testing for antibiotic susceptibility/resistance}

The pattern of antibiotic susceptibility and resistance was identified for each isolate; tests involved the agar diffusion method using Muller-Hinton agar (Oxoid, Basingstoke, UK) against ampicillin $(10 \mu \mathrm{g})$, amoxicillin-clavulanic acid (30 $\mu \mathrm{g})$, cefuroxime $(30 \mu \mathrm{g})$, ceftriaxone $(30 \mu \mathrm{g})$, ceftazidime (30 $\mu \mathrm{g})$, ciprofloxacin $(5 \mu \mathrm{g})$, ofloxacin $(5 \mu \mathrm{g})$, levofloxacin (5 $\mu \mathrm{g})$, meropenem $(10 \mu \mathrm{g})$, erythromycin $(15 \mu \mathrm{g})$, tetracycline (30 $\mu \mathrm{g})$, trimethoprim-sulphamethoxazole $(25 \mu \mathrm{g})$ and chloramphenicol $(30 \mu \mathrm{g})$. MDR was defined as resistance of an isolate to three or more of the antibiotics tested ${ }^{19}$.

\section{Malaria parasite test}

For each participant, we carried out Giemsa staining on both thick and thin blood films for the microscopic identification of malaria parasites. These procedures were carried out in accordance with standard protocols described by the World Health Organization (WHO) ${ }^{25}$.

\section{Data analysis}

Data analysis was carried out using Statistical Package for Social Sciences (SPSS) version 20 (IBM, Chicago, Illinois, United States). Test of normality for the population sample was done using the Kolmogorov-Smirnov's test. Categorical variables are presented as proportions while continuous variables are presented as mean \pm standard deviation (SD) if distributed uniformly. Categorical variables were compared using the chi-square test. Mean values were compared using the Student's t-test while non-normally distributed continuous data were compared using the Kruskal-Wallis test. Sensitivity, specificity, positive predictive value (PPV) and negative predictive value (NPV) were calculated for Widal cut-off titres using culture as the gold standard and a P-value $<0.05$ was considered to be statistically significant.

\section{Results}

The socio-demographic characteristics of the study participants are shown in Table 1. The age of the febrile patients ranged from 12 to 59 years while that of the control group ranged from 12 to 60 years. There was no statistically significant difference between the mean ages of the typhoid

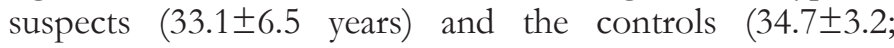

$\mathrm{P}=0.32$ ). There was a marginally higher proportion of females in each of the two groups. The mean duration of febrile illness was 7.6 \pm 3.5 days (range: $1-19$ days).

Table 1. Socio-demographic characteristics of febrile patients and controls

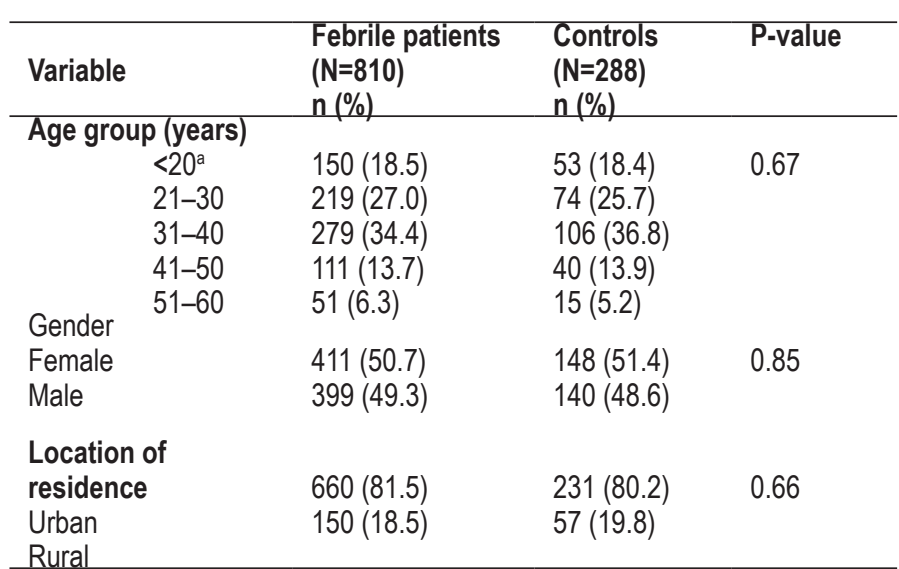

a Age group $<20$ years. For febrile patients, this includes 141 children aged between 12 and 17 years and 9 adults aged between 18 and 19 years. For the controls, this includes 48 children aged between 12 and 17 years and 5 adults aged between 18 and 19 years.

Table 2. Typhoid culture results of febrile patients in Enugu, Nigeria

\begin{tabular}{lllll}
\hline & Blood & Urine & Stool & P-value \\
\hline $\begin{array}{l}\text { Culture result } \\
(\mathrm{N}=810), \mathrm{n}(\%)\end{array}$ & & & & \\
$\begin{array}{l}\text { Positive } \\
\text { Negative }\end{array}$ & $21(2.6)$ & $21(2.6)$ & $72(8.9)$ & $<0.001$ \\
$\begin{array}{l}\text { Isolates } \\
\text { S. paratyphi }(\mathrm{N}=72),\end{array}$ & $6(8.3)$ & $9(12.5)$ & $57(79.2)$ & $<0.001$ \\
$\mathrm{n}(\%)$ & $15(35.7)$ & $12(25.5)$ & $15(35.7)$ & \\
S. typhi $(\mathrm{N}=42), \mathrm{n}(\%)$ & & & & \\
\hline
\end{tabular}

Data relating to the febrile patients with positive cultures are shown in Table 2. Of the 810 patients tested, 114 (14.1\%) were bacteriologically positive for typhoid pathogens. None of the controls had typhoid isolates. Of the 114 patients with positive cultures, S. typhi was isolated in $42(36.8 \%)$ cases while $S$. paratyphi A $(\mathrm{n}=41), \mathrm{B}(\mathrm{n}=20)$, and C $(\mathrm{n}=11)$ strains were isolated from another $72(63.2 \%)$. When data for individual samples were analysed in the 810 typhoid suspects, the rates of culture positivity were as follows: stool $(72 ; 8.9 \%)$, blood $(21 ; 2.6 \%)$ and urine $(21 ; 2.6 \%)$; there was a statistically significant difference in this respect $(\mathrm{P}<0.001)$. When testing individual samples from patients with positive isolates, we found that stool samples yielded predominantly S. paratyphi (79.2\%), while blood and urine samples yielded predominantly $S$. typhi $(35.7 \%$ and $25.5 \%$, respectively); these differences were statistically significant $(\mathrm{P}<0.001)$. Of the 21 typhoid suspects with a positive blood culture result, 7 $(33.3 \%)$ also had a positive stool culture. Febrile patients with a positive culture result were significantly younger $(28.4 \pm 4.6$ years vs $37.7 \pm 8.3$ years, $\mathrm{P}=0.0002)$ and significantly more women had a positive culture result than men (64 [15.6\%] vs 50 [12.5\%]; $\mathrm{P}=0.21)$.

The clinical features of the febrile patients are summarized in Table 3. Only anorexia $(\mathrm{P}=0.02)$, constipation $(\mathrm{P}=0.02)$, abdominal pain/discomfort $(\mathrm{P}<0.001)$, splenomegaly $(\mathrm{P}<0.001)$ and relative bradycardia $(\mathrm{P}<0.001)$ showed statistically significant differences when compared with the clinical features of febrile patients with negative typhoid 


\section{culture results.}

The Widal titres for febrile patients with typhoid fever (confirmed by culture) and febrile patients without typhoid (culture negative) are shown in Tables 4 and 5, respectively. Of the 114 febrile patients with culture-confirmed typhoid fever, 63 (55.3\%) individuals had positive Widal titres for the $\mathrm{O}$ antigen while $6(5.3 \%)$ had positive Widal titres for the $\mathrm{H}$ antigen. Of the 696 febrile patients with negative culture results, $46(6.6 \%)$ and $31(4.5 \%)$ had positive Widal titres for $\mathrm{O}$ and $\mathrm{H}$ antigens, respectively. All 288 of the apparently healthy control subjects had Widal titres $\leq 1: 40$ for $\mathrm{O}$ antigen; only $2(0.69 \%)$ of these subjects had titres of $\geq 1: 160$ for the $\mathrm{H}$ antigen (data not shown).

Table 3. Clinical features of patients evaluated for typhoid fever in Enugu, Nigeria

\begin{tabular}{|c|c|c|c|c|}
\hline Parameter & $\begin{array}{l}\text { Typhoid } \\
\text { suspects } \\
N=810 \\
n(\%)\end{array}$ & $\begin{array}{l}\text { Typhoid } \\
\text { culture [+ve] } \\
N=114 \\
n(\%)\end{array}$ & $\begin{array}{l}\text { Typhoid } \\
\text { culture [-ve] } \\
\mathrm{N}=696 \\
\mathrm{n}(\%)\end{array}$ & P-value \\
\hline Fever & $810(100.0)$ & $114(100.0)$ & $696(100.0)$ & 1.00 \\
\hline Headache & 641 (79.1) & $87(76.3)$ & $554(79.6)$ & 0.42 \\
\hline Anorexia & $553(68.3)$ & $89(78.1)$ & $464(66.7)$ & 0.02 \\
\hline Diarrhoea & $470(58.0)$ & 72 (63.2) & $398(57.2)$ & 0.23 \\
\hline Constipation & $130(16.0)$ & $27(23.7)$ & $104(14.9)$ & 0.02 \\
\hline $\begin{array}{l}\text { discomfort } \\
\text { /pain }\end{array}$ & $432(53.3)$ & $102(89.5)$ & $330(47.4)$ & $<0.001$ \\
\hline Vomiting & $384(47.4)$ & $62(54.4)$ & $322(46.3)$ & 0.12 \\
\hline Pallor & $93(11.5)$ & $11(9.6)$ & $82(11.8)$ & 0.51 \\
\hline Jaundice & $19(2.3)$ & $2(1.8)$ & $17(2.5)$ & 0.65 \\
\hline Hepatomegaly & $125(15.4)$ & $23(20.2)$ & $102(14.7)$ & 0.13 \\
\hline Splenomegaly & $45(5.6)$ & $20(17.5)$ & $25(3.6)$ & $<0.001$ \\
\hline Tachycardia & $404(49.9)$ & $58(50.9)$ & $346(49.7)$ & 0.82 \\
\hline Relative bradycardia & $23(2.8)$ & $14(12.3)$ & $9(1.3)$ & $<0.001$ \\
\hline
\end{tabular}

Table 4. Widal titres of febrile patients with typhoid fever, as confirmed by culture

\begin{tabular}{lllll}
\hline Titre & O-antigen & \multicolumn{3}{c}{$H$-antigen } \\
& Frequency & $\%(\mathrm{~N}=114)$ & Frequency & $\%(\mathrm{~N}=114)$ \\
\hline$<1: 20$ & 15 & 13.2 & 30 & 26.3 \\
$1: 20$ & 6 & 5.3 & 15 & 13.2 \\
$1: 40$ & 30 & 26.3 & 45 & 39.5 \\
$1: 80$ & 15 & 13.2 & 18 & 15.8 \\
$1: 160$ & 36 & 31.6 & 6 & 5.3 \\
$1: 320$ & 6 & 5.3 & 0 & 0 \\
$1: 640$ & 6 & 5.3 & 0 & 0 \\
Total & 114 & 100 & 114 & 100 \\
\hline
\end{tabular}

Of the 810 febrile patients, a total of $180(22.2 \%)$ had malaria parasitaemia, all due to Plasmodium falciparum. Of these 180 patients, $115(63.9 \%)$ had a positive Widal test for $\mathrm{O} / \mathrm{H}$ antigens compared with only $1 \%$ (6/630) for those with negative malaria parasite test results $(\mathrm{P}<0.001)$. However, the frequency of positive typhoid culture results among febrile patients with malaria parasitaemia was not significantly different from that of febrile patients with a negative malaria parasite test (15.6\% vs $13.7 \%, \mathrm{P}=0.52)$. Therefore, $48.3 \%$ of the febrile patients with malaria parasitaemia had a falsepositive Widal test.

Table 5. Widal titres of febrile patients who were negative for typhoid culture

\begin{tabular}{lllll} 
Titre & $\mathrm{O}$ antigen & \multicolumn{3}{c}{$H$ antigen } \\
\cline { 2 - 5 } & Frequency & $\%(\mathrm{~N}=696)$ & Frequency & $\%(\mathrm{~N}=696)$ \\
\hline$<1: 20$ & 546 & 78.4 & 583 & 83.8 \\
$1: 20$ & 60 & 8.6 & 33 & 4.7 \\
$1: 40$ & 44 & 6.3 & 24 & 3.4 \\
$1: 80$ & 40 & 5.7 & 25 & 3.6 \\
$1: 160$ & 6 & 0.9 & 28 & 4.0 \\
$1: 320$ & 0 & 0 & 3 & 0.4 \\
$1: 640$ & 0 & 0 & 0 & 0 \\
Total & 696 & 100 & 696 & 100 \\
\hline
\end{tabular}

Table 6. Sensitivity, specificity, PPV and NPV of titres for anti-O $(\geq 1: 80)$ and anti-H $(\geq 1: 160)$ Widal tests in the diagnosis of typhoid fever among febrile patients in Enugu, Nigeria

\begin{tabular}{llll}
\hline Measurement & $\mathbf{O}$ antigen (\%) & $\begin{array}{l}\text { Hantigen } \\
(\%)\end{array}$ & $\begin{array}{l}\text { Both antigens } \\
(\%)\end{array}$ \\
\hline Sensitivity & 55.3 & 0.9 & 49.1 \\
Specificity & 93.3 & 95.5 & 90.7 \\
PPV & 57.7 & 16.2 & 46.2 \\
NPV & 92.7 & 86.0 & 91.6
\end{tabular}

PPV, positive predictive value; NPV, negative predictive value.

Table 7. Patterns of antibiotic sensitivity/resistance in bacterial isolates among febrile patients with typhoid fever, as confirmed by culture

\begin{tabular}{lllllll}
\hline \multicolumn{5}{c}{ S. typhi(N=42) } & \multicolumn{3}{l}{ S. paratyphi (N=72) } & Total(N=114) \\
\hline Antibiotic & Sensitive & Resistant & Sensitive & Resistant & Sensitive & Resistant \\
& $n(\%)$ & $n(\%)$ & $n(\%)$ & $n(\%)$ & $n(\%)$ & $n(\%)$ \\
\hline Ampicillin & $2(4.8)$ & $40(95.2)$ & $5(6.9)$ & $67(93.1)$ & $7(6.1)$ & $107(93.9)$ \\
Amoxicilin- & $10(23.8)$ & $32(76.2)$ & $15(20.8)$ & $57(79.2)$ & $25(21.9)$ & $89(78.1)$ \\
Clavulanate & $41(97.6)$ & $1(2.4)$ & $68(94.4)$ & $4(5.6)$ & $109(95.6)$ & $5(4.4)$ \\
Ceftriaxone & $33(78.6)$ & $9(21.4)$ & $55(76.4)$ & $17(23.6)$ & $88(77.2)$ & $26(22.8)$ \\
Ceftazidime & $19(45.2)$ & $23(54.8)$ & $19(26.4)$ & $53(73.6)$ & $38(33.3)$ & $76(66.7)$ \\
Cefuroxime & $42(100.0)$ & $0(0.0)$ & $72(100.0)$ & $0(0.0)$ & $114(100.0)$ & $0(0.0)$ \\
Ciprofloxacin & $36(85.7)$ & $6(14.3)$ & $64(88.9)$ & $8(11.1)$ & $100(87.7)$ & $14(12.3)$ \\
Ofloxacin & $22(52.4)$ & $20(47.6)$ & $43(59.7)$ & $29(40.3)$ & $65(57.0)$ & $49(43.0)$ \\
Chloramphenicol & $42(100.0)$ & $0(0.0)$ & $72(100.0)$ & $0(0.0)$ & $114(100.0)$ & $0(0.0)$ \\
Levofloxacin & $42(100.0)$ & $0(0.0)$ & $72(100.0)$ & $0(0.0)$ & $114(100.0)$ & $0(0.0)$ \\
Meropenem & $12(28.6)$ & $30(71.4)$ & $20(27.8)$ & $52(72.2)$ & $32(28.1)$ & $82(71.9)$ \\
Tetracycline & $16(38.1)$ & $26(61.9)$ & $22(30.6)$ & $50(69.4)$ & $38(33.3$ & $76(66.7)$ \\
Co-trimoxazole & $0(0.0)$ & $42(100.0)$ & $0(0.0)$ & $72(100.0)$ & $0(0.0)$ & $114(100.0)$ \\
Erythromycin & & & & & & \\
\hline & & & & & &
\end{tabular}

The sensitivity, specificity, PPV and NPV of the overall positive Widal titre (both antigens) was 49.1\%, 90.7\%, 46.2\% 
and $91.6 \%$, respectively (Table 6 ). The antibiotic susceptibility profile of the typhoid isolates is presented in Table 7. None of the isolates showed resistance to ciprofloxacin, levofloxacin or meropenem. There was only low levels of resistance to ceftriaxone $(4.4 \%)$ and ofloxacin $(12.3 \%)$. However, isolates showed high levels of resistance to erythromycin (100\%), ampicillin (93.9\%), amoxicillin-clavulanate $(78.1 \%)$, tetracycline $(71.9 \%)$, cefuroxime $(66.7 \%)$ and co-trimoxazole $(66.7 \%)$. The patterns of susceptibility for $S$. typhi and S. paratyphi were comparable and the overall rate of antibiotic resistance to three or more agents was $52.6 \%$ (Table 8 ).

Table 8. Patterns of antibiotic multi-drug resistance (MDR) in isolates from febrile patients with typhoid fever, as confirmed by culture

\begin{tabular}{llll}
\hline MDR pattern & $\begin{array}{l}\text { S. typhi } \\
(\mathrm{N}=42)\end{array}$ & $\begin{array}{l}\text { S. paratyphi } \\
(\mathrm{N}=72)\end{array}$ & $\begin{array}{l}\text { Total }(\mathrm{N}=114) \\
\mathrm{n}(\%)\end{array}$ \\
\hline $\mathrm{n}(\%)$ & $\mathrm{n}(\%)$ & \\
R3 & $8(19.0)$ & $18(25.0)$ & $26(22.8)$ \\
R4 & $6(14.3)$ & $6(8.3)$ & $12(10.5)$ \\
R5 & $4(9.5)$ & $10(13.9)$ & $14(12.3)$ \\
R6 & $2(4.8)$ & $4(5.6)$ & $6(5.3)$ \\
R7 & $1(2.4)$ & $1(1.4)$ & $2(1.8)$ \\
TOTAL & $21(50.0)$ & $39(54.2)$ & $60(52.6)$
\end{tabular}

\section{Discussion}

Based on bacteriological evidence, the prevalence of typhoid fever in this study was $14.1 \%$. Previous studies have reported comparable prevalence data of $11.3 \%$ to $18.7 \%$ among febrile patients ${ }^{26-28}$. In one previous study, Maude et al. ${ }^{26}$ reported that $11.3 \%$ of a group of 300 febrile adults/children in Bangladesh were positive for typhoid, as determined by blood culture and polymerase chain reaction (PCR) for $S$. typhi. In another study, involving 1375 febrile patients from all age groups in Nepal, Adhikari et al. found that $17.3 \%$ had typhoid fever based on positive blood culture results for $S$. typh $i^{27}$ while Ramyil et al. reported a prevalence of $18.7 \%$ for typhoid fever among adults/children in North-Central Nigeria, as diagnosed by stool culture ${ }^{28}$. In contrast to our findings, some previous studies reported a higher typhoid prevalence of $22.1 \%$ to $55 \%$ among febrile patients in various settings in Africa and Asia when using blood cultures ${ }^{29-32}$. Other studies in sub-Saharan Africa have reported a lower prevalence of culture-proven typhoid fever, ranging from $1 \%$ to $5 \% \%^{13,19,33-35}$. Beyond the variable epidemiology of typhoid fever across different environments, there are a number of factors that could explain the disparity between our present findings and these earlier studies. For example, a previous study of Indian subjects reported a typhoid fever prevalence as high as $55 \%{ }^{32}$; however, the participants in this study were all children. This was further supported by our own observation that typhoid fever tends to be commoner among younger individuals; a phenomenon that has also been reported by other authors ${ }^{36-38}$. The distribution of gender within the study population may also contribute to the observed differences. For example, a number of studies have reported a male dominance among typhoid fever $\operatorname{cases}^{27,28,36}$, although Chowta et al. reported female dominance ${ }^{39}$. We did not observe any differences between genders among our study population. Sample size could also contribute to the disparity in results between our current study and previous studies. Interestingly, the sample size of our study was 3 to 10 times higher than that of previous studies reporting a higher prevalence of typhoid. It is possible that the previous use of antibiotics by the participants of previous studies could also have affected the prevalence rates.

The type of culture could also influence the observed prevalence of typhoid fever. For example, while several of the existing studies only carried out blood cultures ${ }^{26,27,29-32}$, Elseed et al. cultured blood, urine and stools ${ }^{35}$. In other studies, Gopalakrishnan et al., Nsutebu et al. Eleazar et al. and cultured both blood and stools ${ }^{31,33,40}$ while Ramyil et al. only carried out stool culture ${ }^{28}$. In a retrospective analysis of 130 patients with typhoid fever who presented at a tertiary-care hospital in Tokyo between 1975 and 1998, positive cultures were most frequently found in blood (81\%), followed by stool $(50 \%)$ and urine $(2.1 \%)^{36}$. In a study of Egyptian patients, Youssef et al. found that the rate of recovery of $S$. typhi from blood culture decreased with the duration of symptoms and fell from $72 \%$ in the first week, to $21 \%$ of patients with symptoms in the second week, and to $7 \%$ in patients with symptoms in excess of 15 days $^{41}$. While stools are considered to be less specific than blood for the isolation of pathogens, the performance of simultaneous blood, urine and stool cultures increases the probability of detection. This is considered important in the African setting where late presentation is common; when patients present late it is possible that the blood cultures may not be positive.

Our analysis showed that $S$. paratyphi was more common than $S$. typhi and accounted for $63.2 \%$ of the total number of cases with culture-proven typhoid fever. However, $S$. typhi was the more commonly detected pathogen in blood and urine samples. Generally, $S$. typhi remains the main causative organism of typhoid fever and has been more frequently isolated by blood culture $1,19,27,31,32,34$. Nevertheless, there is evidence to suggest that the incidence of typhoid fever caused by $S$. paratyphi may be increasing ${ }^{33,42,43}$. Furthermore, S. paratyphi tends to cause a milder disease than $S$. typhi and this may partly explain the absence of classical typhoid fever complications in our patients.

The most common clinical features in our patients with positive typhoid cultures were fever, abdominal pain/ discomfort, anorexia, headache, diarrhoea, vomiting and tachycardia. However, only anorexia, constipation, abdominal pain/discomfort, splenomegaly and relative bradycardia showed statistically significant differences when compared with the clinical features of febrile patients with negative typhoid cultures. In accordance with our observations, fever $(90-100 \%)^{1,27,33,36,39,42}$, headache $(50-98 \%)^{1,27,33,42}$ and anorexia $(65-90 \%)^{1,27,33}$ were the most consistent findings among typhoid fever patients in other settings. Although diarrhoea $(19-56 \%)^{27,33,36,39,42}$, abdominal pain $(11-48 \%)^{27,33,39,42}$ and vomiting $(20-46 \%)^{27,33,42}$ were commonly reported buy other studies, the proportions of individuals with these symptoms were lower than that seen in our current study.

Though the confirmatory diagnosis of typhoid fever is by the isolation of $S$. typhi or $S$. paratyphi from appropriate samples, most developing countries, such as Nigeria, have continued to depend on the Widal test, largely due to limited laboratory facilities for culture and more modern tests based on nucleic acids. In the present study, the overall sensitivity, specificity, PPV and NPV of the Widal test were $49.1 \%$, $90.7 \%, 46.2 \%$ and $91.6 \%$, respectively. The low sensitivity of the Widal test shows that the test has only low ability to correctly identify individuals who actually have typhoid fever and therefore does not represent a good test for screening. 
The PPV represents the proportion of patients with a disease that are correctly identified. Hence, our findings suggest that a positive Widal test would have a low predictive value for the presence of typhoid fever and as such would be a poor diagnostic test. Specificity is the probability that a truly infected individual will test positive while NPV is the probability that those testing negative are truly non-infected. Accordingly, our data suggest that a negative Widal test may be useful for excluding typhoid fever in the differential diagnosis of acute febrile illness. This may be helpful in tropical countries since such regions are associated with a plethora of diseases that can cause acute febrile illness. A major challenge in the comparison of findings across different studies is the variable cut-off titres for a positive Widal reaction. In agreement with our findings, Adhikari et al. showed that a Widal test performed on acute phase serum in febrile patients in an endemic population in Nepal had low sensitivity $(45.2 \%)$ and PPV $(34.2 \%)$ but high specificity $(82.3 \%)$ and NPV $(87.8 \%)^{27}$. Another study, conducted in Egypt, indicated that a negative Widal test result would have a good predictive value $(\mathrm{NPV}=98 \%)$ while a positive result would have a poor predictive value $(\mathrm{PPV}=57 \%)^{40}$. The poor diagnostic performance of a single Widal test in endemic regions has been demonstrated in other studies ${ }^{13,19,33,34,44}$ In contrast to these observations, two studies carried out in endemic settings in India (PPV=91\%, NPV=31\%) and Bangladesh (PPV=81.1\%, NPV $=31.7 \%$ ) found that a single Widal test was a relevant diagnostic tool for typhoid fever ${ }^{32,45}$ One of these previous studies found that the cut-off titre for a positive test was 1:80 for both $\mathrm{O}$ and $\mathrm{H}$ antigens ${ }^{32}$. Beyond this, it is possible that the relatively small sample size of these earlier studies may partly account for their observations.

Our present study identified malaria parasitaemia in one-fifth of the typhoid suspects, suggesting that malaria is a more common cause of acute febrile illness in our environment. A high proportion of individuals with malaria parasitaemia had positive Widal reactions, a phenomenon that has been observed in other studies ${ }^{29,33}$. The co-existence of malaria parasitaemia and culture-proven typhoid fever was only seen in $15.6 \%$ of our patients, which showed that $48 \%$ of the patients with malaria parasitaemia would have been misdiagnosed of typhoid fever if we had relied on the Widal test alone. In a previous study, Enabulele et al. reported a co-infection of typhoid fever and malaria in $20 \%$ of febrile Nigerian patients and demonstrated a statistically significant association between the severity of malaria parasitaemia and a positive Widal test $\mathrm{t}^{29}$. False-positive Widal titres among febrile patients with malaria parasitaemia have been previously documented and are thought to be due to the cross-reactivity of the antigen ${ }^{46}$. It is possible that the malaria parasite Plasmodium may share similar strong immunogenic antigens with the typhoid organism $S$. typhi. Infection by $P$. falciparum could therefore evoke the production of antibodies against antigens that will cross-react with $S$. typhi antigens in the test kit, thus leading to a false positive result. This theory is supported by the strong correlation observed between malaria parasite loading and Salmonella antibody titres in previous studies ${ }^{29,46}$. A heightened anamnestic response among febrile parasitaemic patients, thus leading to the production of antibodies that can bind strongly to shared similar antigens, could also contribute to false positive Widal titres in febrile patients with malaria parasitaemia. These findings suggest that the indiscriminate use of the Widal test could lead to the over-diagnosis of typhoid fever and the unnecessary use of antibiotics in patients with malaria.

The over-prescription of antibiotics for typhoid fever in developing countries with weak antibiotic policies, based on clinical suspicion and/or a single Widal test, poses a significant risk for the emergence of antibiotic resistance. One of the earliest studies conducted in SouthWest Nigeria (20 years ago) demonstrated high levels of resistance (ranging from $51 \%$ to $91 \%$ ) of typhoid isolates to commonly used agents (ampicillin, chloramphenicol, cotrimoxazole, tetracycline and cefotaxime) but showed 100\% susceptibility to ciprofloxacin and ofloxacin, which had been newly developed at that time ${ }^{47}$. Another report from NorthCentral Nigeria, more than 10 years later, showed evidence of MDR for $S$. typhi involving both old and new drugs48; however, the exact burden of MDR was not quantified in this previous study. Previous studies in Africa also confirmed a changing pattern of antibiotic resistance and MDR in typhoid isolates ${ }^{49,50}$. This highlights the need for a periodic update of the pattern of antibiotic susceptibility for typhoid isolates.

Our study showed high levels of resistance (22.8$100 \%$ ) for typhoid isolates to several older and relatively newer antibiotics. Our patients showed no resistance to ciprofloxacin, levofloxacin and meropenem while only $4.4 \%$ showed resistance to ceftriaxone; $52.6 \%$ of our patients showed resistance to three or more antibiotic agents. Our findings are in agreement with previous studies from subSaharan Africa which found MDR in $50 \%$ and $52 \%$ of typhoid isolates in Ethiopia and Ghana, respectively19,51. A large population-based study in Kenya found that S. typhi isolates had a much higher MDR of $77.8 \%{ }^{52}$. On the other hand, Jamil et al. reported MDR in 36\% of typhoid pathogens in Pakistan ${ }^{42}$. In a surveillance study carried out in Asia, Ochiai et al. demonstrated considerable geographic variation in the proportion of typhoid isolates that were MDR within the same region ${ }^{37}$, thus making a strong case for local evidencebased antibiotic guidelines. The wide distribution and growing prevalence of MDR among typhoid isolates has led to fluoroquinolones assuming a primary role in the treatment of typhoid fever. However, over time, the widespread use of these agents has been associated with reduced susceptibility and documented resistance in some populations ${ }^{53,54}$. Despite this growing concern, the findings of our present study suggest that ciprofloxacin, ofloxacin and ceftriaxone can be sustained as first-line antibiotic options for typhoid fever in our setting. Levofloxacin and carbapenems would be better reserved for cases that are resistant to the current first-line options.

To the best of our knowledge, this is one of the most recent attempts to characterize the burden of MDR in the typhoid pathogens of Nigeria. A major strength of our study was the large sample size. Our data were also more robust than in other studies because we carried out cultures simultaneously on blood, stools and urine samples. Another strength is the inclusion of levofloxacin and meropenem; these drugs are being increasingly prescribed on an empirical basis, although their effectiveness has been rarely assessed in local studies. Nevertheless, this study had some limitations which need to be considered. First, we were unable to exclude whether our patients were carriers of Salmonella; thus, it is difficult to verify whether some febrile patients with stool isolates were just carriers. Moreover, stools are not sterile. Consequently, we might only be able to refer to these patients as possible cases 
of typhoid fever cases despite the bacteriological evidence. Although we made efforts to select our control group from the relatives of the cases with typhoid, this proved to be difficult. Thus, we had a good number of controls that were not related to the typhoid suspects. In addition, among the controls who were relatives of the typhoid cases, some were adults that lived in separate homes from the typhoid cases. These demographic differences probably explain why the Widal titres in our control group were not higher than in the general population. Another limitation of this study was that we were unable to perform Widal titres on samples from convalescents. Although some patients had follow-up clinic visits (ranging from 1 to 4 weeks after their initial visit), it was difficult to get Widal tests performed on these samples from convalescent patients. We excluded individuals who reported the previous use of antibiotics. Antibiotic abuse is fairly common in developing countries; as such, we were careful to limit the impact of previous antibiotic use on culture results. However, we recognize the fact it might not be entirely accurate to depend upon patient reports to exclude individuals with a history of recent antibiotic use. Although we used measured body temperature as an inclusion criterion for fever, rather than a history of fever, in order to increase the sensitivity of our blood culture surveillance, it is possible that this practice could potentially have led to a higher rate of culture-positive typhoid suspects in this study. On the other hand, it is also possible that we could have missed some typhoid cases with body temperatures that were lower than our threshold. At the time of this study, only an adult blood culture system was available in our hospital. As a result, we were only able to include children who were between 12 and 17 years of age. Children less than 12 years were not included because we could not acquire a culture medium system with a small enough volume for paediatric cases.

\section{Conclusion}

In conclusion, this study found that culture positivity among typhoid fever suspects was common. Salmonella enterica serovar paratyphi was more commonly isolated than $S$. enterica serovar Typhi. The Widal agglutination test performed poorly as a diagnostic test. We also observed that malaria was a more common cause of febrile illness and was associated with a high proportion of false-positive Widal titres. The prevalence of antibiotic MDR was high and predominantly affected older drugs and a few of the newer agents. We suggest that ciprofloxacin and ceftriaxone can be sustained as first-line antibiotic options for typhoid fever while levofloxacin and carbapenems would be better reserved for cases that are resistant to the current first-line options. There is a clear need for a periodic review of the antibiotic susceptibility of typhoid pathogens.

\section{Authors' contributions (roles)}

MEO, HCG and UM designed the study, UM, MEO and ODO supervised data collection, MOI, MEO and ODO performed data analysis and drafted the manuscript, HCG, MOI and MEO critically revised the manuscript for intellectual content. All authors read and approved the final manuscript.

\section{Funding}

No funding was received for this research.

Availability of data and materials: Available.

\section{Competing interests}

The authors have no competing interests.

\section{References}

1. Mitra R, Houshang MA, Hamid HS, Maryam D, Reza MA, Shima H, et al. Clinical features of patients with typhoid fever and drug resistance of the causative isolates in western Iran. Trop Doct. 2009;39(4):223-4. DOI: $10.1258 / \mathrm{td} .2009 .090046$.

2. Azmatullah A, Qamar FN, Thaver D, Zaidi AK, Bhutta ZA. Systematic review of the global epidemiology, clinical and laboratory profile of enteric fever. J Glob Health. 2015;5(2):020407. DOI: 10.7189/ jogh.05.020407.

3. Woods CW, Murdoch DR, Zimmerman MD, Glover WA, Basnyat B, Wolf L, et al. Emergence of Salmonella enterica serotype Paratyphi A as a major cause of enteric fever in Kathmandu, Nepal. Trans R Soc Trop Med Hyg. 2006;100:1063-67. DOI: 10.1016/j.trstmh.2005.12.011.

4. Mogasale V, Maskery B, Ochiai RL, Lee JS, Mogasale VV, Ramani $\mathrm{E}$, et al. Burden of typhoidfever in low-income and middle-income countries: a systematic, literature-based update with risk-factor adjustment. Lancet Glob Health. 2014; 2(10):e570-80. DOI: 10.1016/ S2214-109X (14)70301-8.

5. Crump JA, Luby SP, Mintz ED. The global burden of typhoid fever. Bull World Health Organ. 2004;82:346-53.

6. Buckle GC, Walker CL, Black RE. Typhoid fever and paratyphoid fever: systematic review to estimate global morbidity and mortality for 2010. J Glob Health. 2012;2:010401. DOI: 10.7189/jogh.02.010401.

7. von Kalckreuth V, Konings F, Aaby P, Adu-Sarkodie Y, Ali M, Aseffa A, et al. The Typhoid Fever Surveillance in Africa Program (TSAP): Clinical, diagnostic, and epidemiological methodologies. Clin Infect Dis. 2016;62(1):9-16. DOI: 10.1093/cid/civ693.

8. Antillón M, Warren JL, Crawford FW, Weinberger DM, Kürüm E, Pak GD, et al. The burden of typhoid fever in low-and-middleincome countries: A meta-regression approach. PLoS Negl Trop Dis. 2017;11(2):e0005376. DOI: 10.1371/journal.pntd.0005376.

9. GBD 2017 Typhoid and Paratyphoid Collaborators. The global burden of typhoid and paratyphoid fevers: a systematic analysis for the Global Burden of Disease Study 2017. Lancet Infect Dis. 2019;19(4):369-81. DOI: 10.1016/S1473-3099(18)30685-6.

10. World Health Organization (WHO). Vaccine Preventable disease surveillance standards- Typhoid and other invasive salmonellosis. Last updated 2018 Sep [cited 2019 Jun 08]. Available from: https://www. who.int/immunization/monitoring_surveillance/burden/vpd/WHO_ SurveillanceVaccinePreventable_21_Typhoid.

11. Wain J, Hosoglu S. The laboratory diagnosis of enteric fever. J Infect Dev Ctries. 2008;2(6):421-5.

12. Olopoenia LA, King AL. Widal agglutination test -100 years later: still plagued by controversy. Postgrad Med J. 2000;76:80-4. DOI: 10.1136/pmj.76.892.80.

13. Ley B, Mtove G, Thriemer K, Thriemer K, Amos B, Seidlein LV, et al. Evaluation of the Widal tube agglutination test for the diagnosis of typhoid fever among children admitted to a rural hospital in Tanzania and a comparison with previous studies. BMC Infect Dis. 2010;10:180. DOI: $10.1186 / 1471-2334-10-180$.

14. Bhutta ZA. Current concepts in the diagnosis and treatment of typhoid fever. Br Med J. 2006;333:78-82. DOI: 10.1136/bmj.333.7558.78.

15. Willke A, Ergonul O, Bayar B. Widal test in diagnosis of typhoid fever in Turkey. Clin Diagn Lab Immunol. 2002;9:938-41. DOI: 10.1128/CDLI.9.4.938-941.2002.

16. Olsen SJ, Pruckler J, Bibb W, Thanh NT, Trinh T, Minh NT, et al. Evaluation of rapid diagnostic tests for typhoid fever. J Clin Microbiol. 2004;42(5):1885-9. DOI: 10.7860/JCDR/2016/20426.8684.

17. Ngwu BA, Agbo JA. Typhoid fever: clinical diagnosis versus 
laboratory confirmation. Niger J Med. 2003;12:187-92.

18. Khoharo HK, Ansari S, Qureshi F. Evaluating single acutephase Widal test for the diagnosis of typhoid fever. Med Channel. 2010;16(1):42-4.

19. Wasihun AG, Wlekidan LN, Gebremariam SA, Welderufael AL, Muthupandian S, Haile TD, et al. Diagnosis and treatment of typhoid fever and associated prevailing drug resistance in northern Ethiopia. Int J Infect Dis. 2015;35:96-102. DOI: 10.1016/j.ijid.2015.04.014.

20. Enugu State Ministry of Health. Strategic Health Dev Plan 20102015. Enugu, Nigeria.

21. National Population Commission. Nigeria Demographic and Health Survey (NDHS) 2013. Abuja, Nigeria.

22. Baron EJ, Finegold SM. Bailey and Scotts Diagnostic Microbiology 8th ed. St Louis: Mosby; 1990. pp. 197-212.

23. Hawkey PM, Lewis DA. Medical bacteriology, a practical approach. 1st ed. Oxford: Oxford University Press; 198. pp. 21-42.

24. Freter R. Agglutination titration (Widal) for the diagnosis of enteric fever and other entero bacterial infections. In: Rose NR, Friedman H, editors. Manual of clinical immunology. Washington D.C.: American Society for Microbiology; 1976.

25. World Health Organization. Bench aids for the diagnosis of malaria infections. Geneva: WHO; 2000.

26. Maude RR, Ghose A, Samad R, de Jong HK, Fukushima M, Wijedoru L, et al. A prospective study of the importance of enteric fever as a cause of non-malarial febrile illness in patients admitted to Chittagong medical college hospital, Bangladesh. BMC Infect Dis. 2016;16:567. DOI: 10.1186/s12879-016-1886-3.

27. Adhikari A, Rauniyar R, Raut PP, Manadhar KD, Gupta BP. Evaluation of sensitivity and specificity of ELISA against Widal test for typhoid diagnosis in endemic population of Kathmandu. BMC Infect Dis. 2015;15:523. DOI: 10.1186/s12879-015-1248-6.

28. Ramyil, MS, Ihuoma, OJ, Ogundeko TO, Ameh JM, Olorundare F, Adeniyi OG, et al. Comparative study on the use of Widal test and stool culture in the laboratory diagnosis of Salmonella infection in adult and children in Jos metropolis, Plateau State, Nigeria. Int J Sci Res. 2013;2(12):435-41.

29. Enabulele O, Awunor SN. Typhoid fever in a tertiary hospital in Nigeria: another look at the Widal agglutination test as a preferred option for diagnosis. Niger Med J. 2016;57:145-9. DOI: 10.4103/03001652.184057 .

30. Keddy KH, Sooka A, Letsoalo ME, Hoyland G, Chaignat CL, Morrissey AB, et al. Sensitivity and specificity of typhoid fever rapid antibody tests for laboratory diagnosis at two sub-Saharan African sites. Bull World Health Organ. 2011;11:1-15.

\section{DOI: 10.2471/BLT.11.087627.}

31. Gopalakrishnan V, Sekhar WY, Soo EH, Vinsent RA, Devi S. Typhoid fever in Kuala Lumpur and a comparative evaluation of two commercial diagnostic kits for the detection of antibodies to Salmonella typhi. Singapore Med J 2002;43:354-8.

32. Aziz T, Haque SS. Role of Widal test in the diagnosis of typhoid fever in context to other test. Am J Biochem Biotechnol. 2012;2:16-18. DOI: $10.2471 /$ blt.06.039818.

33. Nsutebu EF, Martins P, Adiogo D. Prevalence of typhoid fever in febrile patients with symptoms clinically compatible with typhoid fever in Cameroon. Trop Med Int Health. 2003;8 (6):575-8. DOI: 10.12691/ ajmr-3-6-1.

34. Andualem G, Abebe T, Kebede N, Gebre-Selassie S, Mihret A, Alemayehu H. A comparative study of Widal test with blood culture in the diagnosis of typhoid fever in febrile patients. BMC Res Notes. 2014;7:653. DOI: 10.1186/1756-0500-7-653.

35. Elseed YH. Comparison between the Widal test and culturing technique in the diagnosis of enteric fever in Khartoum State, Sudan. Afr J Bacteriol Res. 2015;7(5):56-9. DOI: 10.9734/BMRJ/2015/17986.

36. Hoshino Y, Masuda G, Negishi M, Ajisawa A, Imamura A, Hachimori K, et al. Clinical and bacteriological profiles of patients with typhoid fever treated during 1975-1998 in the Tokyo metropolitan Komagome hospital. Microbiol Immunol. 2000;44(7):577-83. DOI: 10.1111/j.1348-0421.2000.tb02536.x.

37. Ochiai RL, Acosta CJ, Danovaro-Holliday MC, Baiqing D, Bhattacharya SK. A study of typhoid fever in five Asian countries: disease burden and implications for controls. Bull World Health Organ. 2008;86:260-68. DOI: 10.2471/blt.06.039818.

38. Ezeigbo OR, Agomoh NG, Asuoha-Chuks N. Laboratory diagnosis of typhoid fever using Widal and blood culture methods in Aba, Southeastern Nigeria. Am J Microb Res. 2015;3(6):181-3. DOI: 10.12691/ajmr-3-6-1.

39. Chowta MN, Chowta NK. Study of clinical profile and antibiotic response in typhoid fever. Indian J Med Microbiol. 2005;23 (2):125-7. DOI: $10.4103 / 0255-0857.16054$.

40. Eleazar CI, Iroegbu CU, Udoh IP. Comparison of Widal test with cultural methods for evaluation of Salmnellatyphi/paratyphi infection. Br Microb Res J. 2015;8(5):577-84. DOI: 10.1136/jcp.36.4.471.

41. Youssef FG, Daba AS, Kabeil SS, Parker TM. A comparative study of bloodculture and antibody response with the duration of illness in diagnosis of typhoid fever. Aust J Basic Appl Sci. 2010;4(4):609-14. DOI: 10.1515/znc-2000-5-630.

42. Jamil B, Lashari I, Bhatti S, Hasan RS. Enteric fever in adult patients: clinical features, outcome and antibiotic susceptibility patterns. Infect Dis J Pak. 2004;13:68-72.

43. Versa G, Jaspal K, Chander J. An increase in enteric fever cases due to Salmonella paratyphi A in and around Chandigarh. Indian J Med Res. 2009;129:95-8.

44. Omuse G, Kohli R, Revathi G. Diagnostic utility of a single Widal test in the diagnosis of typhoid fever at Aga Khan university hospital (AKUH), Nairobi, Kenya. Trop Doct. 2010;40(1):43-4. DOI: 10.1258/ td.2009.090109.

45. Alam AS, Rupam FA, Chaiti F. Significance and value of the Widal test in the diagnosis of typhoid fever. Bangladesh J Child Health 2011;35:53-8.

46. Ajero CMU, Uzoagba DC. Antigenic cross reaction between malaria parasites and typhoid organisms in patients with fever of unknown origin in Owerri municipal Imo state. Niger Journal of Microbiol. 2015;28:2892-901.

47. Akinyemi KO, Coker AO, Olukoya DK, Oyefolu AO, Amorighoye EP, Omonigbehin EO. Prevalence of multi-drug resistant Salmonella typhi among clinically diagnosed typhoid fever patients in Lagos, Nigeria. Z Naturforsch C. 2000;55 (5-6):489-93. DOI: 10.1515/znc2000-5-630.

48. Adabara NU, Ezugwu BU, Momoijimoh A, Madzu A, Hashimu Z, Damisa D. The prevalence and antibiotic susceptibility pattern of Salmonella typhi among patients attending a military hospital in Minna, Nigeria.Adv Prev Med. 2012;2012:875419. DOI: 10.1155/2012/875419.

49. Wasfy MO, Frenck R, Ismail TF, Mansour H, Malone JL, Mahoney FJ. Trends of multiple-drug resistance among Salmonella typhi isolates during a 14-year period in Egypt. Clin Infect Dis. 2002;35(10):1265-6. DOI: $10.1086 / 343052$.

50. Kariuki S, Revathi G, Muyodi J, Mwituria J, Munyalo A, Mirza S, et al. Characterization of multidrug-resistant typhoid outbreaks in Kenya. J Clin Microbiol. 2004;42(4):1477-82. DOI: 10.1128/jcm.42.4.14771482.2004 .

51. Mills-Robertson F, Addy ME, Mensah P, Crupper SS. Molecular 
characterization of antibiotic resistance in clinical Salmonella typh isolated in Ghana. FEMS Microbiol Lett. 2002;215 (2):249-53. DOI: 10.1111/j.1574-6968.2002.tb11398.x.

52. Breiman RF, Cosmas L, Njuguna H, Audi A, Olack B, Ochieng JB, et al. Population-based incidence of typhoid fever in an urban informal settlement and a rural area in Kenya: implications for typhoid vaccine use in Africa. PLoS One. 2012;7(1):e29119. DOI: 10.1371/journal. pone.0029119.
53. Joshi S, Amamath SK. Fluoroquinolone resistance in Salmonella typhi and S. paratyphi A in Bangalore, India. Trans Roy Soc Trop Med Hyg. 2007;101(3):308-10. DOI: 10.1016/j.trstmh.2006.05.009.

54. Saleh FOI, Ahmed HA, Khairy RMM, Abdelwahab SF. Increased quinolone resistance among typhoid Salmonella isolated from Egyptian patients. J Infect Dev Ctries. 2014;8(5):661-5. DOI: 10.3855/jidc.4111. 